doi:10.13108/2021-13-1-137

\title{
ON ASYMPTOTIC STRUCTURE OF CONTINUOUS-TIME MARKOV BRANCHING PROCESSES ALLOWING IMMIGRATION WITHOUT HIGHER-ORDER MOMENTS
}

\author{
A.A. IMOMOV, A.Kh. MEYLIEV
}

Dedicated to our Parents

\begin{abstract}
We consider a continuous-time Markov branching process allowing immigration. Our main analytical tool is the slow variation (or more general, a regular variation) conception in the sense of Karamata. The slow variation property arises in many issues, but it usually remains rather hidden. For example, denoting by $p(n)$ the perimeter of an equilateral polygon with $n$ sides inscribed in a circle with a diameter of length $d$, one can check that the function $\boldsymbol{\pi}(n):=p(n) / d$ converges to $\pi$ in the sense of Archimedes, but it slowly varies at infinity in the sense of Karamata. In fact, it is known that $p(n)=d n \sin (\pi / n)$ and then it follows $\boldsymbol{\pi}(\lambda x) / \boldsymbol{\pi}(x) \rightarrow 1$ as $x \rightarrow \infty$ for each $\lambda>0$. Thus, $\boldsymbol{\pi}(x)$ is so slowly approaching $\pi$ that it can be suspected that " $\pi$ is not quite constant".

Application of Karamata functions in the branching processes theory allows one to bypass severe constraints concerning existence of the higher-order moments of the infinitesimal characteristics of the process under study. Zolotarev was one of the first who demonstrated an encouraging prospect of application of the slow variation conception in the theory of Markov branching processes and has obtained principally new results on asymptote of the survival probability of the process without immigration.

In this paper, delving deeply in the nature of the Karamata functions, we study more subtle properties of branching processes allowing immigration. In particular, under quite admissible conditions, we find explicit forms for the generating functions of invariant measures for the process under consideration.
\end{abstract}

Keywords: Markov branching process, immigration, transition functions, state space classification, generating functions, slowly varying function, invariant measures.

Mathematics Subject Classification: 60J80; 26A12

\section{INTRODUCTION AND MAIN RESULTS}

1.1. Background, aim and main assumptions. We deal with the model of population growth called continuous-time Markov branching process allowing immigration (MBPI). This process can have a simple physical interpretation: a population size changes not only as a result of reproduction and disappearance of existing individuals, but also at the random stream of inbound "extraneous" individuals of the same type from outside. The population of individuals evolves as follows. Each individual existing at time $t \in \mathcal{T}:=[0,+\infty)$ independently of his history and of each other for a small time interval $(t, t+\varepsilon)$ transforms into $j \in \mathbb{N}_{0} \backslash\{1\}$ individuals with probability $a_{j} \varepsilon+o(\varepsilon)$ and, with probability $1+a_{1} \varepsilon+o(\varepsilon)$, stays to live or makes evenly one descendant as $\varepsilon \downarrow 0$, where $\mathbb{N}_{0}=\{0\} \cup \mathbb{N}$ and $\mathbb{N}$ is the set of natural numbers.

A.A. Imomov, A.Kh. Meyliev, On asymptotic structure of Continuous-time Markov BranchING PROCESSES ALLOWING IMMIGRATION WITHOUT HIGHER-ORDER MOMENTS.

(C) A.A. Imomov, A.Kh. Meyliev. 2021.

Submitted June 20, 2020. 
Here $\left\{a_{j}\right\}$ are the intensities of individuals transformation such that $a_{j} \geqslant 0$ for $j \in \mathbb{N}_{0} \backslash\{1\}$ and $0<a_{0}<-a_{1}=\sum_{j \in \mathbb{N}_{0} \backslash\{1\}} a_{j}<\infty$. Independently of these processes, for each time interval $j \in \mathbb{N}$, new individuals leave the population with a probability $b_{j} \varepsilon+o(\varepsilon)$ and the immigration does not occur with a probability $1+b_{0} \varepsilon+o(\varepsilon)$. The immigration intensities $b_{j} \geqslant 0$ for $j \in \mathbb{N}$ and $0<-b_{0}=\sum_{j \in \mathbb{N}} b_{j}<\infty$. Newly arrived individuals undergo transformation in accordance with the reproduction law generated by the intensities $\left\{a_{j}\right\}$, see [12, Ch. VII, Sect. 1]. Thus, the process under consideration is completely determined by infinitesimal generating functions

$$
f(s)=\sum_{j \in \mathbb{N}_{0}} a_{j} s^{j} \quad \text { and } \quad g(s)=\sum_{j \in \mathbb{N}_{0}} b_{j} s^{j} \quad \text { for } \quad s \in[0,1) .
$$

Let $X(t)$ be the population size at a time $t \in \mathcal{T}$ in MBPI. This is a homogenous continuoustime Markov chain with the state space $\mathcal{S} \subset \mathbb{N}_{0}$ and transition functions

$$
p_{i j}(t):=\mathbb{P}_{i}\{X(t)=j\}=\mathbb{P}\{X(t+\tau)=j \mid X(\tau)=i\}
$$

for all $i, j \in \mathcal{S}$ and $\tau, t \in \mathcal{T}$. An appropriate probability generating function, see [5],

$$
\mathcal{P}_{i}(t ; s):=\sum_{j \in \mathcal{S}} p_{i j}(t) s^{j}=(F(t ; s))^{i} \exp \left(\int_{0}^{t} g(F(u ; s)) d u\right),
$$

where $F(t ; s)$ is generating function of $Z(t)$ is the Markov branching process initiated by a single particle without immigration components.

It is known that the classification of the state space $\mathcal{S}$ depends on a value of the parameter $m:=f^{\prime}(1-)$. According to the general classification of continuous-time Markov chains, the process $X(t)$ is called subcritical, critical, and supercritical if respectively $m<0, m=0$ and $m>0$, see [12, Ch VII, Sect. 2].

We consider the critical case only. In this case, Sevastyanov [1] proved that if $2 b:=f^{\prime \prime}(1-)$ is finite and the immigration law has a finite mean, then the normalized process $X(t) / b t$ has the limiting Gamma distribution function $\Gamma_{1, \lambda}(x), x \geqslant 0$, where $\lambda=g^{\prime}(1-) / b$. Pakes [9] proved that $t^{\lambda} \mathcal{P}_{i}(t ; s)$ converges to $\pi(s)$ as $t \rightarrow \infty$ and this limit had the power series expansion and generated an invariant measure $\left\{\pi_{j}, j \in \mathcal{S}\right\}$ for MBPI if and only if

$$
\sum_{j \in \mathbb{N}} a_{j} j^{2} \ln j<\infty \quad \text { and } \quad \sum_{j \in \mathbb{N}} b_{j} j \ln j<\infty .
$$

According an appropriate result in paper [7], the invariant measure of MBPI can also be constructed by the strong ratio limit property of transition functions. Namely, the set of positive numbers $\left\{v_{j}:=\lim _{t \rightarrow \infty} p_{0 j}(t) / p_{00}(t)\right\}$ is an invariant measure. We mention a close relation between the sequences $\left\{\pi_{j}, j \in \mathcal{S}\right\}$ and $\left\{v_{j}, j \in \mathcal{S}\right\}$, and accordingly between generating functions $\pi(s)$ and $\mathcal{U}(s)=\sum_{j \in \mathcal{S}} v_{j} s^{j}$. In fact, these are just different versions of the same limit law. It is easy to see that $\mathcal{U}(s)=\pi(s) / \pi(0)$, and this is consistent with uniqueness, up to a multiplicative constant, of the invariant measure of MBPI.

Among various problems relating to asymptotic properties of process states, the one is of an exceptional interest; this problem is on asymptotic representation of $\mathcal{P}_{i}(t ; s)$ and on finding an explicit form for generating function of the invariant measures of MBPI. This problem was first formulated and solved in [9]. In paper [5], a significant improvement in the results of [9] was made. The results of the mentioned papers held under higher-order moment conditions, namely, as $f^{\prime \prime \prime}(1-)<\infty$ and $g^{\prime \prime}(1-)<\infty$.

The main aim of this paper is to improve the results from [5] on finding an explicit form for the generating function of invariant measures omitting the finiteness conditions for the higherorder moments of $f(s)$ and $g(s)$. For this, we will substantially use the approaches of the theory 
of regularly varying functions in the sense of Karamata, see, for instance, [2] and [10]. We recall that a function $L(x)$ is called slowly varying at infinity $\left(\mathbf{S V}_{\infty}\right)$ if it is defined on $(0, \infty)$, positive and

$$
\lim _{x \rightarrow \infty} \frac{L(\lambda x)}{L(x)}=1
$$

for each $\lambda>0$. The representation theorem states that each $\mathbf{S V}_{\infty}$-function may be written in the form

$$
L(x)=c(x) \exp \left(\int_{a}^{x}\left(\frac{\varepsilon(u)}{u}\right) d u\right)
$$

for some $a>0$, where $c(x)$ is a bounded function such that $c(x) \rightarrow c>0$ and $\varepsilon(x)$ is a continuous function such that $\varepsilon(x) \rightarrow 0$ as $x \rightarrow \infty$. If $c(x) \equiv c$ then $L(x)$ is said to be the normalized $\mathbf{S V}_{\infty}$. A function $V(x)$ is called regularly varying at infinity $\left(\mathbf{R V}_{\infty}\right)$ with index $\rho$ if it is defined on $(0, \infty)$ and

$$
\lim _{x \rightarrow \infty} \frac{V(\lambda x)}{V(x)}=\lambda^{\rho}
$$

for all $\lambda>0$. This is why each $\mathbf{R V}_{\infty}$-function can be expressed as $V(x)=x^{\rho} L(x)$ for some $L(x)$ being an $\mathbf{S V}_{\infty}$-function.

We make the following assumptions on $f(s)$ and $g(s)$ :

$$
f(s)=(1-s)^{1+\nu} \mathcal{L}\left(\frac{1}{1-s}\right)
$$

and

$$
g(s)=-(1-s)^{\delta} \ell\left(\frac{1}{1-s}\right)
$$

for all $s \in[0,1)$, where $0<\nu, \delta<1$ and functions $\mathcal{L}(\cdot), \ell(\cdot)$ are $\mathbf{S V}_{\infty}$. By the criticality of our process, the assumption $\left[f_{\nu}\right]$ implies $2 b:=f^{\prime \prime}(1-)=\infty$. If $b<\infty$ then $\left[f_{\nu}\right]$ holds with $\nu=1$ and $\mathcal{L}(t) \rightarrow b$ as $t \rightarrow \infty$. Similarly, a generating function $g(s)$ of the form $\left[g_{\delta}\right]$ generates the law of immigrants arrival having the moment of $\delta$-order. If $g^{\prime}(1-)<\infty$ then $\left[g_{\delta}\right]$ holds with $\delta=1$ and $\ell(t) \rightarrow g^{\prime}(1-)$ as $t \rightarrow \infty$.

Throughout the paper $\left[f_{\nu}\right]$ and $\left[g_{\delta}\right]$ will be our main assumptions.

We make also some extra assumptions for $\mathcal{L}(x)$ and $\ell(x)$. Namely, we assume that

$$
\frac{\mathcal{L}(\lambda x)}{\mathcal{L}(x)}=1+\mathcal{O}(\alpha(x)) \quad \text { as } \quad x \rightarrow \infty
$$

for each $\lambda>0$, where $\alpha(x)$ is some given positive decreasing function so that $\alpha(x) \rightarrow 0$ as $x \rightarrow \infty$. In this case $\mathcal{L}(x)$ is called $\mathbf{S} \mathbf{V}_{\infty}$ with remainder $\alpha(x)$ in the sense of condition SR1 [2, Ch III, $§ 12.1$, condition SR1]. Wherever we employ the condition $\left[\mathcal{L}_{\nu}\right]$, we suppose in addition that

$$
\alpha(x)=\mathcal{O}\left(\frac{\mathcal{L}(x)}{x^{\nu}}\right) \quad \text { as } \quad x \rightarrow \infty .
$$

Similarly, we also assume the condition

$$
\frac{\ell(\lambda x)}{\ell(x)}=1+\mathcal{O}(\beta(x)) \quad \text { as } \quad x \rightarrow \infty
$$

for each $\lambda>0$, where

$$
\beta(x)=\mathcal{O}\left(\frac{\ell(x)}{x^{\delta}}\right) \quad \text { as } \quad x \rightarrow \infty .
$$


1.2. Main results. It is known that $F(t ; s) \rightarrow q$ as $t \rightarrow \infty$ uniformly in $s \in[0,1)$, where $q$ is an extinction probability of the process (see [12, Theorem 3, Ch II, §1]) and in our case $q=1$ (see [12, Theorem 4, Ch II, $\S 1]$ ). Then it is sufficient to consider an asymptote of $\mathcal{P}(t ; s):=\mathcal{P}_{0}(t ; s)$. Then under the above main assumptions, and due to the Kolmogorov backward equation $\partial F / \partial t=f(F)$, it follows from equation (1.1) that

$$
\mathcal{P}(t ; s)=\exp \left(\int_{s}^{F(t ; s)} \frac{g(x)}{f(x)} d x\right) \rightarrow U(s) \quad \text { as } \quad t \rightarrow \infty,
$$

where the limit function $U(s)$ is of the form

$$
U(s)=\exp \left(-\int_{s}^{1}(1-x)^{\gamma-1} \mathrm{~L}\left(\frac{1}{1-x}\right) d x\right)
$$

and $\gamma=\delta-\nu$,

$$
\mathrm{L}(x):=\frac{\ell(x)}{\mathcal{L}(x)} .
$$

In what follows, depending on the sign of $\gamma$, we consider three possible cases concerning the classification of the state space $\mathcal{S}$. It is clear that integral in $(1.2)$ converges if $\gamma>0$ and it diverges if $\gamma<0$. And as it was shown in [7], $\mathcal{S}$ is positive-recurrent if $\gamma>0$, and it is transient if $\gamma<0$. The special case $\gamma=0$ implies that $g(s)=f^{\prime}(s)$ and that $\mathrm{L}(t) \rightarrow 1+\nu$ as $t \rightarrow \infty$. Then we get another population process called Markov Q-process instead of MBPI. We refer to [4] and [6] for the details on the Markov Q-process; see also [1, Ch I, Part D, §14] and [8] for the discrete-time case.

We denote

$$
\tau(t):=\frac{(\nu t)^{1 / \nu}}{\mathcal{N}(t)}
$$

where a function $\mathcal{N}(x)$ is $\mathbf{S V}_{\infty}$ such that

$$
\mathcal{N}^{\nu}(t) \mathcal{L}\left(\frac{(\nu t)^{1 / \nu}}{\mathcal{N}(t)}\right) \rightarrow 1 \quad \text { as } \quad t \rightarrow \infty
$$

Our first theorem describes asymptotic properties of transition function $p_{00}(t)$.

Theorem 1.1. Let the main assumptions be satisfied and $\gamma \neq 0$.

(i) If $\gamma>0$, then $\lim _{t \rightarrow \infty} p_{00}(t)=$ constant. More precisely, there exists a $\mathbf{S V}_{\infty}$-function $L_{0}(t)$ such that $L_{0}(t) L^{-1}(t) \rightarrow 1$ and

$$
-\ln p_{00}(t)=\frac{1}{\gamma} L_{0}(1)(1+o(1)) \quad \text { as } \quad t \rightarrow \infty .
$$

(ii) If $\gamma<0$, then

$$
-(\tau(t))^{-|\gamma|} \ln p_{00}(t)=\frac{1}{|\gamma|} L(\tau(t))(1+o(1)) \quad \text { as } \quad t \rightarrow \infty .
$$

Our second theorem provides an explicit form of generating function of invariant measures depending on the sign of the parameter $\gamma$.

Theorem 1.2. Let the main assumptions be satisfied and $\gamma>0$. Then the limit $U(s)=\lim _{t \rightarrow \infty} \mathcal{P}(t ; s)$ is well-defined and is of the form of (1.2). The function $U(s)$ is finite 
on $s \in[0,1]$ and its representation by a power series $U(s)=\sum_{j \in \mathcal{S}} u_{j} s^{j}$ generates an invariant distribution $\left\{u_{j}\right\}$ with respect to transition functions $\left\{p_{i j}(t)\right\}$. If, in addition to the main assumptions, conditions $\left[\mathcal{L}_{\nu}\right]$ and $\left[\ell_{\delta}\right]$ are satisfied, then

$$
U(s)=\exp \left(-\frac{1}{\gamma} \frac{g(s)}{\Lambda(1-s)}(1+\mathcal{O}(\Lambda(1-s)))\right) \quad \text { as } \quad s \uparrow 1,
$$

where $\Lambda(y)=y^{\nu} \mathcal{L}(1 / y)$.

In the case $\gamma<0$, asymptotic formula 1.5 suggests that we should find a limit of the function $e^{T(t)} \mathcal{P}(t ; s)$ as $t \rightarrow \infty$ with

$$
T(t)=(\tau(t))^{|\gamma|} .
$$

An asymptotic representation for $\mathrm{L}(t)$ will also be of need. In accordance with slowly varying theory, $\ell(\cdot)$ and $\mathcal{L}(\cdot)$ are positive. Moreover, by virtue of [2, Ch III, $\S 12.1$, Corollary 3.12.3], we write the following assertions:

- $\left[\mathcal{L}_{\nu}\right] \quad \Longleftrightarrow \quad \mathcal{L}(t)=C_{\mathcal{L}}+\mathcal{O}(\alpha(t)) \quad$ as $\quad t \rightarrow \infty$,

- $\left[\ell_{\delta}\right] \Longleftrightarrow \ell(t)=C_{\ell}+\mathcal{O}(\beta(t)) \quad$ as $\quad t \rightarrow \infty$,

where $C_{\mathcal{L}}, C_{\ell}$ are positive constants and functions $\alpha(x)$ and $\beta(x)$ are in $\left[\mathcal{L}_{\nu}\right]$ and $\left[\ell_{\delta}\right]$. We then can show that

$$
\mathrm{L}(t)=\frac{\ell(t)}{\mathcal{L}(t)}=C_{\mathrm{L}}+\mathcal{O}\left(\frac{1}{t^{\delta}}\right) \quad \text { as } \quad t \rightarrow \infty
$$

where $C_{\mathrm{L}}=C_{\ell} / C_{\mathcal{L}}$. Especially, this result is sharp in the case of $C_{\mathrm{L}}=|\gamma|$. In view of Theorem 1.1 (ii), we obtain the following result.

Theorem 1.3. Let $\gamma<0$ and conditions $\left[\mathcal{L}_{\nu}\right]$ and $\left[\ell_{\delta}\right]$ be satisfied. If $\nu<2 \delta$ and $C_{L}=|\gamma|$ in (1.7), then the following asymptotic relation holds:

$$
e^{T(t)} \mathcal{P}(t ; s)=\pi(s)(1+\rho(t ; s)),
$$

where $\rho(t ; s) \rightarrow 0$ as $t \rightarrow \infty$ uniformly in $s \in[0, d], d<1$, and the limiting generating function $\pi(s)$ reads as

$$
\pi(s)=\exp \left(\frac{1}{(1-s)^{|\gamma|}}+\int_{s}^{1}\left(\frac{g(u)}{f(u)}+\frac{|\gamma|}{(1-u)^{1+|\gamma|}}\right) d u\right) .
$$

Its power series representation $\pi(s)=\sum_{j \in \mathcal{S}} \pi_{j} s^{j}$ generates an invariant measure $\left\{\pi_{j}\right\}$ for the process $X(t)$.

Remark 1. We observe that the statement of Theorem 1.3 is compatible with the results of papers [9] and [5] established for the case of $\max \left\{f^{\prime \prime}(1-), g^{\prime}(1-)\right\}<\infty$. Our theorem essentially strengthens last-mentioned results. Under the assumptions of Theorem 1.3, the right-hand side in (1.5) tends to 1.

Remark 2. The conditions $C_{L}=|\gamma|$ and $\nu<2 \delta$ in Theorem 1.3 are essential because they ensure the convergence of the integral in (1.9). In fact, due to the main assumptions and relation (1.7), an expression $(1-u)^{\mu-1}$ majorizes the integrand, where $\mu:=2 \delta-\nu>0$. Therefore, the function

$$
\mathcal{B}(s):=\exp \left(\int_{s}^{1}\left(\frac{g(u)}{f(u)}+\frac{|\gamma|}{(1-u)^{1+|\gamma|}}\right) d u\right)
$$


is bounded for $s \in[0, d], d<1$. Hence,

$$
\ln \pi(s) \sim \frac{1}{(1-s)^{|\gamma|}} \quad \text { as } \quad s \uparrow 1 .
$$

Corollary. Under the assumptions of Theorem 1.3, the identity

$$
e^{T(t)} p_{00}(t)=\mathcal{B}(0)(1+o(1)) \quad \text { as } \quad t \rightarrow \infty
$$

holds, where the function $\mathcal{B}(s)$ is defined in (1.10).

\section{AUXILIARIES}

In this section we provide a series of auxiliary statements, which will serve as key ingredients in the proof of our main results.

First we are interested in asymptotic expansion of generating function of Markov branching processes (MBP) without immigration. Let $F(t ; s)$ is generating function of MBP initiated by single individual. The following lemma describing an asymptotic representation of the function $R(t ; s):=1-F(t ; s)$, was proved in [4] (see also [3]). We formulate it in a slightly different form more convenient for our purposes.

Lemma 2.1. If condition $\left[f_{\nu}\right]$ holds then

$$
\frac{1}{R(t ; s)}=\frac{(\nu t)^{1 / \nu}}{\mathcal{N}(t)}\left[1+\frac{\mathcal{M}(s)}{t}\right]^{1 / \nu}
$$

for all $s \in[0,1)$, where the function $\mathcal{N}(x)$ is $\mathbf{R V}_{\infty}$ defined in (1.3) and $\mathcal{M}(s)$ is the generating function of invariant measures of MBP having the form

$$
\mathcal{M}(s)=\int_{1}^{1 /(1-s)} \frac{d x}{x^{1-\nu} \mathcal{L}(x)} .
$$

The asymptotic behaviour of integrals of $\mathbf{R V}_{\infty}$-functions will be employed in proof of our results. Let $L_{0}(t)$ be normalized $\mathbf{S V}_{\infty}$-function defined on $[0, \infty)$ and consider a function $V(x)=x^{\rho} L_{0}(x)$, where $\rho=-(1+\sigma) \neq-1$. For each $c \geqslant 1$ we define an integral

$$
\mathcal{J}(t):=\int_{c}^{t} V(y) d y=\int_{c}^{t} y^{-(1+\sigma)} L_{0}(y) d y .
$$

An improper integral $\mathcal{J}:=\lim _{t \rightarrow \infty} \mathcal{J}(t)$ converges as $\sigma>0$, and it diverges as $\sigma \leqslant 0$.

We first consider the case $\sigma>0$ and we are going to find this integral explicitly. By integration by parts we obtain:

$$
\mathcal{J}(t)=-\left.\frac{1}{\sigma} \frac{L_{0}(y)}{y^{\sigma}}\right|_{c} ^{t}+\frac{1}{\sigma} \int_{c}^{t} y^{-\sigma} d\left[L_{0}(y)\right] .
$$

Since $L_{0}(t)$ is normalized $\mathbf{S} \mathbf{V}_{\infty}$, it can be represented as follows:

$$
L_{0}(y)=C \exp \int_{a}^{y} \frac{\varepsilon(t)}{t} d t
$$

for some $C>0$ and $a>0$, where $\varepsilon(t) \rightarrow 0$ as $t \rightarrow \infty$. Differentiating the above formula, we find that

$$
d\left[L_{0}(y)\right]=L_{0}(y) \frac{\varepsilon(y)}{y} d y
$$


and (2.3) yields

$$
\int_{c}^{t} y^{-(1+\sigma)} L_{0}(y)\left(1-\frac{1}{\sigma} \varepsilon(y)\right) d y=-\left.\frac{1}{\sigma} \frac{L_{0}(y)}{y^{\sigma}}\right|_{c} ^{t} .
$$

We define an $\mathbf{S V}_{\infty}$-function $L(\cdot)$ as follows:

$$
L(t):=L_{0}(t)\left(1-\frac{1}{\sigma} \varepsilon(t)\right) .
$$

Then

$$
L_{0}(t) L^{-1}(t)=1+\mathcal{O}(\varepsilon(t)) \quad \text { as } \quad t \rightarrow \infty .
$$

Now formula (2.5) leads us to the following lemma on the finiteness the integral $\mathcal{J}(t)$.

Proposition. Let $L(t)$ be a normalized $\mathbf{S V}_{\infty}$-function and $\sigma>0$. Then there exists a function $L_{0}(t)$ with the same properties as $L(t)$ such that $L_{0}(t) L^{-1}(t) \rightarrow 1$ as $t \rightarrow \infty$ and the identity

$$
\int_{c}^{t} y^{-(1+\sigma)} L(y) d y=\frac{1}{\sigma}\left[\frac{1}{c^{\sigma}} L_{0}(c)-\frac{1}{t^{\sigma}} L_{0}(t)\right]
$$

holds true for each $c \geqslant 1$.

Below we provide an example of finding the function $L_{0}(t)$ in (2.6). Assume that $L(t)=\ln (a t+b)$ for some positive constants $a$ and $b$. In this case, integration by parts gives:

$$
\int_{c}^{t} y^{-(1+\sigma)} \ln (a y+b) d y=-\left.\frac{1}{\sigma} \frac{\ln (a y+b)}{y^{\sigma}}\right|_{c} ^{t}+\frac{1}{\sigma} J(t),
$$

where

$$
J(t)=\int_{c}^{t} \frac{a d y}{a y^{1+\sigma}+b y^{\sigma}} .
$$

It is easy to confirm that

$$
0 \leqslant \frac{a}{a+b} \frac{1}{\sigma}\left[\frac{1}{c^{\sigma}}-\frac{1}{t^{\sigma}}\right] \leqslant J(t) \leqslant \frac{1}{\sigma}\left[\frac{1}{c^{\sigma}}-\frac{1}{t^{\sigma}}\right]
$$

and hence

$$
J(t)=\theta \frac{1}{\sigma}\left[\frac{1}{c^{\sigma}}-\frac{1}{t^{\sigma}}\right],
$$

where $\theta$ is a positive constant such that $a(a+b)^{-1} \leqslant \theta \leqslant 1$. Using the latter expression for $J(t)$ in (2.7), we arrive easily to (2.6) with a required $\mathbf{S V}_{\infty}$-function $L_{0}(t)$ reading as

$$
L_{0}(t)=\ln (a t+b)+\theta \frac{1}{\sigma}, \quad \text { where } \quad \frac{a}{a+b} \leqslant \theta \leqslant 1 .
$$

In particular, if $L(t)=\ln t$, then letting $a=1$ and $b=0$, we get $\theta=1$ and

$$
L_{0}(t)=\ln t+\frac{1}{\sigma} .
$$

Let us estimate the decay rate for the remainder $\mathcal{J}(\infty)-\mathcal{J}(t)$. 
Lemma 2.2. Let $L(t)$ be a $\mathbf{S V}_{\infty}$-function and $\sigma>0$. Then

$$
\int_{t}^{\infty} y^{-(1+\sigma)} L(y) d y=\frac{1}{\sigma} \frac{1}{t^{\sigma}} L(t)(1+\varrho(t))
$$

where $\varrho(t) \rightarrow 0$ as $t \rightarrow \infty$. In addition, if $L(t)$ is a $\mathbf{S V}_{\infty}$-function with remainder $r(t)$, then $\varrho(t)=\mathcal{O}(r(t))$ as $t \rightarrow \infty$.

Proof. Making the change of variables $y=u t$, we find that

$$
I(t):=\int_{t}^{\infty} y^{-(1+\sigma)} L(y) d y=\frac{L(t)}{t^{\sigma}}\left(\int_{1}^{\infty} u^{-(1+\sigma)} d u+\int_{1}^{\infty}\left(\frac{L(u t)}{L(t)}-1\right) u^{-(1+\sigma)} d u\right) .
$$

The first integral can be found explicitly:

$$
\int_{1}^{\infty} u^{-(1+\sigma)} d u=\frac{1}{\sigma}
$$

The expression in brackets of the second integrand is bounded thanks to Potter theorem [2, Ch I, §5.4, Theorem 1.5.6] and tends to 0 as $t \rightarrow \infty$ uniformly in $u \in(0,1]$. Hence,

$$
I(t)=\frac{L(t)}{t^{\sigma}}\left(\frac{1}{\sigma}+\varrho(t)\right)
$$

where $\varrho(t) \rightarrow 0$ as $t \rightarrow \infty$. If $L(x)$ is a $\mathbf{S V}_{\infty}$-function with remainder $r(t)$, then the expression in the brackets expression tends to 0 with the same rate, and hence $\varrho(t)=\mathcal{O}(r(t))$. This implies (2.8) and completes the proof.

We consider the case $\sigma<0$; we stress that we do not need to consider the case $\sigma=0$. Since in the considered case the integral $\mathcal{J}(t)$ diverges, we need to estimate the main part of its asymptotics.

Lemma 2.3. Let $L(t)$ be an $\mathbf{S V}_{\infty}$-function and $\sigma<0$. Then

$$
\int_{c}^{t} y^{-(1+\sigma)} L(y) d y=\frac{1}{|\sigma|} t^{|\sigma|} L(t)(1+o(1)) \quad \text { as } \quad t \rightarrow \infty
$$

for each $c \geqslant 1$.

We omit the proof of this lemma because it reproduces literally the of Lemma 2.2 and also an alternative proof was given in [2, Ch I, Sect. 5.6, Prop. 1.5.8].

\section{Proof of MAIN RESUlts}

This section is devoted to the proof of our main results.

Proof of Theorem 1.1. Letting $i=0, s=0$ in (1.1) and using the Kolmogorov backward equation $\partial F / \partial t=f(F)$, we find that

$$
\begin{aligned}
p_{00}(t) & =\exp \left(\int_{0}^{t} g(F(u ; 0)) d u\right)=\exp \left(\int_{0}^{F(t ; 0)} \frac{g(x)}{f(x)} d x\right) \\
& =\exp \left(-\int_{0}^{F(t ; 0)}(1-u)^{\gamma-1} \mathrm{~L}\left(\frac{1}{1-u}\right) d u\right)=\exp \left(-\int_{1}^{1 / R(t)} y^{-(1+\gamma)} \mathrm{L}(y) d y\right),
\end{aligned}
$$


where $R(t)=R(t ; 0)$. Since $R(t) \rightarrow 0$, the integral in 3.1 tends to $\int_{1}^{\infty} y^{-(1+\gamma)} \mathrm{L}(y) d y$ as $t \rightarrow \infty$, which is finite obviously. Hence, the $\operatorname{limit}_{t \rightarrow \infty} \lim _{00}(t)$ is finite. In order to prove (1.4), we use Proposition 2 with $c=1$ in (3.1). Then the function $\mathrm{L}(y)$ is necessary normalized. We let $\Lambda(y):=f(1-y) / y$ for $y \in(0,1]$. Then $\Lambda(y)=y^{\nu} \mathcal{L}(1 / y)$ and it was shown in [2, Ch VIII, §12.3] that

$$
\frac{y \Lambda^{\prime}(y)}{\Lambda(y)}=\nu+o(1) \quad \text { as } \quad y \downarrow 0 .
$$

Integrating this relation shows, that $\mathcal{L}(y)$ is normalized. In same way we can justified that $\ell(y)$ is also normalized. Hence, the function $\mathrm{L}(y)=\ell(y) / \mathcal{L}(y)$ is also normalized. Now representation (2.2) for the function $\mathcal{M}(s)$ from Lemma 2.1 implies that it is bounded for $s \in[0, d], d<1$ and $\mathcal{\mathcal { M }}(0)=0$. Therefore, relation (2.1) yields that $\tau(t)=R^{-1}(t)$. Hence, it follows from 2.6) and (3.1) that there exists a function $\mathrm{L}_{0}(t)$ such that $\mathrm{L}_{0}(t) \mathrm{L}^{-1}(t) \rightarrow 1$ and

$$
-\ln p_{00}(t)=\int_{1}^{1 / R(t)} y^{-(1+\gamma)} \mathrm{L}(y) d y \sim \frac{1}{\gamma}\left(\mathrm{L}_{0}(1)-(\tau(t))^{\gamma} \mathrm{L}_{0}(\tau(t))\right)
$$

as $t \rightarrow \infty$. Last term in $(3.2)$ tends to zero since $\tau(t) \rightarrow 0$.

Using Lemma 2.3, by (3.1) we obtain:

$$
-\ln p_{00}(t)=\frac{1}{|\gamma|} \frac{1}{(R(t))^{|\gamma|}} \mathrm{L}_{0}(\tau(t))(1+o(1)) \quad \text { as } \quad t \rightarrow \infty .
$$

Since $\mathrm{L}_{0}(t) \mathbf{L}^{-1}(t) \rightarrow 1$ and $\tau(t)=R^{-1}(t)$, the latter relation is equivalent to 1.5 . The proof is complete.

Proof of Theorem 1.2. It is clear that $U(s)=\lim _{t \rightarrow \infty} \mathcal{P}(t ; s)$ is of form (1.2) and it is finite on $s \in[0,1]$. It is known that $F(t+\tau ; s)=F(t ; F(\tau ; s))$; see [9, p. 134]. Then

$$
\begin{aligned}
\mathcal{P}(t+\tau ; s) & =\mathcal{P}(\tau ; s) \cdot \exp \left(\int_{\tau}^{t+\tau} g(F(u ; s)) d u\right) \\
& =\mathcal{P}(\tau ; s) \cdot \exp \left(\int_{0}^{t} g(F(u ; F(\tau ; s))) d u\right)=\mathcal{P}(\tau ; s) \cdot \mathcal{P}(t ; F(\tau ; s)),
\end{aligned}
$$

and taking limit as $t \rightarrow \infty$, we get the following Schröder type functional equation:

$$
U(F(\tau ; s))=\frac{1}{\mathcal{P}(\tau ; s)} U(s) \quad \text { for each } \quad \tau \in \mathcal{T} .
$$

By the power series expansion $U(s)=\sum_{j \in \mathcal{S}} u_{j} s^{j}$, equation $\sqrt{3.3}$ implies an invariant property $u_{j}=\sum_{i \in \mathcal{S}} u_{i} p_{i j}(\tau)$ for each $\tau>0$. Moreover, it is easy to see that $U(1-)$. Thus, $\left\{u_{j}, j \in \mathcal{S}\right\}$ is an invariant distribution for MBPI.

We substitute $y=(1-x)^{-1}$ into $(1.2)$ and we obtain its alternative form:

$$
U(s)=\exp \left(-\int_{1 /(1-s)}^{\infty} y^{-(1+\gamma)} \mathrm{L}(y) d y\right) .
$$


Using Lemma 2.2, the integral in (3.4) becomes

$$
\int_{1 /(1-s)}^{\infty} y^{-(1+\gamma)} \mathrm{L}(y) d y=\frac{1}{\gamma}(1-s)^{\gamma} \mathrm{L}\left(\frac{1}{1-s}\right)\left(1+r\left(\frac{1}{1-s}\right)\right)
$$

as $s \uparrow 1$. It is easy to see that due to assumptions $\left[\mathcal{L}_{\nu}\right]$ and $\left[\ell_{\delta}\right]$ the remainder term of $\mathrm{L}(t)$ satisfies $r(t)=\mathcal{O}\left(\mathcal{L}(t) / t^{\nu}\right)$ for $\gamma>0$. Then owing to $\Lambda(y)=y^{\nu} \mathcal{L}(1 / y)$, the representation 1.6$)$ is implied by (3.4) and (3.5). The proof is complete.

Proof of Theorem 1.3. As above, we have:

$$
\begin{aligned}
e^{T(t)} \mathcal{P}(t ; s) & =\exp \left((\tau(t))^{|\gamma|}+\int_{0}^{t} g(F(u ; s)) d u\right) \\
& =\exp \left(\Delta(t ; s)+(\tau(t ; s))^{|\gamma|}+\int_{s}^{F(t ; s)} \frac{g(x)}{f(x)} d x\right),
\end{aligned}
$$

where

$$
\tau(t ; s)=R^{-1}(t ; s) \quad \text { and } \quad \Delta(t ; s)=(\tau(t))^{|\gamma|}-(\tau(t ; s))^{|\gamma|} .
$$

We confirm easily that

$$
(\tau(t ; s))^{|\gamma|}=\frac{1}{(1-s)^{|\gamma|}}+\int_{s}^{F(t ; s)} \frac{|\gamma|}{(1-u)^{1+|\gamma|}} d u .
$$

Along with this we see that $\tau(t)=\tau(t ; 0)$. Therefore, considering $\mathcal{M}(0)=0$, by representation (2.1) we have

$$
\begin{aligned}
\Delta(t ; s) & =(\tau(t))^{|\gamma|}\left(1-\left(1+\frac{\mathcal{M}(s)}{t}\right)^{|\gamma| / \nu}\right) \\
& \sim-|\gamma|(\tau(t))^{|\gamma|} \frac{\mathcal{M}(s)}{\nu t}=-|\gamma| \frac{\mathcal{M}(s)}{(\nu t)^{\delta / \nu \mathcal{N}|\gamma|}(t)} \quad \text { as } \quad t \rightarrow \infty .
\end{aligned}
$$

Since the function $\mathcal{M}(s)$ is bounded for $s \in[0, d], d<1$, the above relation implies that

$$
\Delta(t ; s)=\mathcal{O}\left(\frac{\mathcal{L}_{\gamma}(t)}{t^{\delta / \nu}}\right) \rightarrow 0 \quad \text { as } \quad t \rightarrow \infty
$$

where $\mathcal{L}_{\gamma}(t)=\mathcal{N}^{-|\gamma|}(t)$. Combining relations $(3.6),(3.7),(3.8)$, we get

$$
e^{T(t)} \mathcal{P}(t ; s)=\exp \left(\frac{1}{(1-s)^{|\gamma|}}+\mathcal{A}(t ; s)+o(1)\right) \quad \text { as } \quad t \rightarrow \infty,
$$

where

$$
\mathcal{A}(t ; s):=\int_{s}^{F(t ; s)}\left(\frac{g(u)}{f(u)}+\frac{|\gamma|}{(1-u)^{1+|\gamma|}}\right) d u .
$$

Since $F(t ; s) \rightarrow 1$, the right-hand side of (3.9) tends to $\pi(s)$ defined in (1.9) as $t \rightarrow \infty$ uniformly in $s \in[0, d], d<1$. Hence, identity 1.8 holds.

Finally, we see that the function $\pi(s)$ satisfies equation (3.3). Therefore, denoting its power series representation by $\pi(s)=\sum_{j \in \mathcal{S}} \pi_{j} s^{j}$, we arrive at the invariant property $\pi_{j}=\sum_{i \in \mathcal{S}} \pi_{i} p_{i j}(\tau)$ for each $\tau>0$. Thus, $\left\{\pi_{j}, j \in \mathcal{S}\right\}$ is an invariant measure for the Markov branching process $X(t)$. The proof is complete. 
Proof of Corollary. The statement is immediately obtained from relation (1.8) by letting $x=$ 0 .

\section{ACKNOWLEDGEMEnTs}

The authors are deeply grateful to the anonymous referee for his careful reading of the manuscript, for valuable suggestions and comments.

\section{BIBLIOGRAPHY}

1. K.B. Athreya, P.E. Ney. Branching processes. Springer, New York (1972).

2. N.H. Bingham, C.M. Goldie and J.L. Teugels. Regular variation. Cambridge Univ. Press, Cambridge (1987).

3. A.A. Imomov, A.Kh. Meyliyev. On application of slowly varying functions with remainder in the theory of Markov branching processes with mean one and infinite variance // Ukrainian Math. $\mathrm{J}$, to appear.

4. A.A. Imomov. On conditioned limit structure of the Markov branching process without finite second moment // Malaysian J. Math. Sci. 11:3, 393-422 (2017).

5. A.A. Imomov. On long-term behavior of continuous-time Markov branching processes allowing immigration // J. Siberian Federal Univ. Math. Phys. 7:4, 443-454 (2014).

6. A.A. Imomov. On Markov analogue of Q-processes with continuous time // Theory Prob. Math. Stat. 84, 57-64 (2012).

7. J. Li, A. Chen, A.G. Pakes. Asymptotic properties of the Markov branching process with immigration // J. Theor. Prob. 25:1, 122-143 (2012).

8. A.G. Pakes. Revisiting conditional limit theorems for the mortal simple branching process // Bernoulli, 5:6, 969-998 (1999).

9. A.G. Pakes. On Markov branching processes with immigration // Sankhyā: The Indian J. Stat. A37, 129-138 (1975).

10. E. Seneta. Regularly varying functions. Springer, Berlin (1976).

11. B.A. Sevastyanov, Limit theorems for stochastic branching processes of special form // Teor. Veroyat. Pril. 2:3, 339-348 (1957). [Theory Probab. Appl. 2:3, 321-331 (1957).]

12. B.A. Sevastyanov. Branching processes. Nauka, Moscow (1971). (in Russian).

13. V.M. Zolotarev. More exact statements of several theorems in the theory of branching processes // Teor. Veroyat. Pril. 2:2, 256-266 (1957). [Theory Probab. Appl. 2:2, 245-253 (1957).]

Imomov Azam Abdurakhimovich,

Department of Mathematics,

Karshi State University,

17, Kuchabag street,

180100 Karshi city, Uzbekistan

E-mail: imomov_azam@mail.ru

Meyliev Abror Khujanazarovich,

Department of Mathematics,

Karshi State University,

17, Kuchabag street,

180100 Karshi city, Uzbekistan

E-mail: abror_meyliyev@mail.ru 